\title{
Introduction to the Best Papers of the ACM Multimedia Systems (MMSys) Conference 2018 and the ACM Workshop on Network and Operating System Support for Digital Audio and Video (NOSSDAV) 2018 and the International Workshop on Mixed and Virtual Environment Systems (MMVE) 2018
}

The 9th ACM International Conference on Multimedia Systems (MMsys 2018) took place in Amsterdam, The Netherlands, from June 12 to 15, with the co-located workshops: 10th International Workshop on Immersive Mixed and Virtual Environment Systems (MMVE2018), 16th Annual Workshop on Network and Systems Support for Games (NetGames2018), 23rd Packet Video Workshop (PV2018), and 28th Annual Workshop on Network and Systems Support for Digital Audio and Video (NOSSDAV2018).

MMSys 2018 introduced some novelties and extended previous successful initiatives of the MMSys series with the main objectives to:

- promote open science by supporting the datasets and open source track, and by granting ACM reproducibility badges;

- increase diversity and facilitate inclusion by offering many travel grants to young, diverse scholars; and

- increase the impact and broaden the community by incorporating top-quality workshops and forward-looking special sessions.

The special sessions focussed on key emerging topics, such as human-centric internet and multimedia systems; immersive multimedia experiences; integrative computer vision and multimedia systems; IoT and smart cities; and multimedia in 5G network architectures. A record number of MMsys papers, 31 (45\% of all the accepted articles), obtained an ACM badge by making data sets and code available, which will allow other researchers to reproduce the results presented in these papers. We acknowledge the generous assistance of ACM SIGMM, which supported a large number of young scholars (21 in total) to attend MMSys via two types of travel grants: one for students with an accepted article and another one for nurturing new talent in SIGMM (part of the SIGMM Funding for New Initiatives including Desk Study of Gender and Diversity within SIGMM). We are delighted to see that MMSys has become a venue that is of high interest to the research community. This was expressed by a total of 115 submissions ( 56 for the research track and 59 combined for the special sessions). Out of the 115 submissions, a total of 30 papers were accepted for publication. This represents a $26 \%$ acceptance rate. As in previous years, a high standard was applied for the review process. For the research track, each paper received a minimum of four reviews, while for the special sessions, each paper received at least three reviews. Authors also had the opportunity to rebut the reviews, and decisions were taken based on the reviews, the rebuttal, and the discussion between the reviewers. Obviously, establishing such a rigorous, double-blind review process would not have been possible without the tireless efforts of the program committee.

The ACM MMSys'18 Technical Program Committee (TPC) co-chairs invited the authors of highquality papers from the research track, special sessions, and workshops to submit their extended

(c) 2019 Copyright held by the owner/author(s).

1551-6857/2019/07-ART64

https://doi.org/10.1145/3339846 
manuscripts to this TOMM special section. After several rounds of thorough reviews, mainly on the new materials beyond the conference publications required by ACM TOMM, we accepted four submissions in this special section.

The first article, by Bentaleb et al., presents a theoretical analysis of adaptive bitrate streaming and demonstrates the benefits of Game Theory for adaptive video selection and network resource allocation. In the article entitled "Game of Streaming Players: Is Consensus Viable or an Illusion?", the authors adopt established game designs to the problem at hand to underpin the design of a game-theory-based quality adaptation algorithm for ABR streaming. The MMSys 2018 submission by the authors received the best student paper award.

The second article, by Chen and Elzarki, entitled "A Framework for Adaptive Residual Streaming for Single Player Cloud Gaming," extended the best workshop paper award submission. The original work was submitted to MMVE 2018. The extended workshop article presents a collaborative rendering framework between the server and the client for 3D game services capable of shifting computational workload toward the edge. It proposes a unique approach to tackle Cloud gaming scalability and latency problems.

The third article, entitled "From Theory to Practice: Improving Bitrate Adaptation in the DASH Reference Player," by Spiteri et al., secured a best paper honourable mention during MMSys 2018. The article presents interesting solutions to improve playback qualities for different videostreaming applications. Two ABR algorithms are proposed with significant improvement on the conventional throughput- and buffer-based algorithms and are now parts of the official DASH reference player. The FAST SWITCHING algorithm is a very practical solution for enhancing the performance of DASH-based adaptive streaming.

Finally, "6K and 8K Effective Resolution with 4K HEVC Decoding Capability for OMAFcompliant 360r Streaming," by Zare et al., provides us with a novel method for packing 360-degree video with $6 \mathrm{k}$ or $8 \mathrm{k}$ effective resolution at the viewport, compatible with $4 \mathrm{k}$ decoders. The key concept is to pick high resolution video around the viewing zone and low-resolution video in other zones and then arrange different zones into a $4 \mathrm{k}$ format. The MMSys 2018 submission was awarded the best student workshop paper award.

We hope you will enjoy the special section. We sincerely thank the authors of all the articles in this special section for their tremendous efforts in preparing and polishing the manuscripts. Furthermore, and in particular, we are grateful to the reviewers of the special section for their valuable and constructive comments throughout the rigorous review process. Without their great help, we would not be able to get the special section ready. Last, we are grateful to ACM TOMM editorial members Professor Alberto Del Bimbo and Professor Stefano Berretti for their generous support. Hopefully, this special section will provide some more momentum on further growing the ACM Multimedia Systems community.

Pablo Cesar

Centrum Wiskunde \& Informatica

Michael Zink

University of Massachusetts in Amherst

Niall Murray

Athlone Institute of Technology

Guest Editors 